Mini review

\title{
Energy Storage Device Application Based on MXenes Composites: a Mini Review
}

\author{
Jun Lv*, Qinghua Huang, Tiejun Liu and Qiaoyu Pan \\ School of Intelligent Manufacturing, Zhejiang Guangsha Vocational and Technical University of \\ Construction, No. 1 Guangfu East Road, Dongyang City, Zhejiang Province, 322100, P.R. China \\ *E-mail: junlv@vtcuni.com
}

doi: $10.20964 / 2021.04 .13$

Received: 8 December 2020 / Accepted: 15 January 2021 / Published: 28 February 2021

With the rapid development of wearable electronic products, increasing attention has been given to flexible energy storage devices. MXenes are a kind of two-dimensional graphene material discovered in recent years. This material has ultra-high volume specific capacity, metal-level conductivity, good hydrophilicity and rich surface chemistry, so it has been widely used in flexible energy storage electrode materials. MXene materials also have some shortcomings that need to be solved, such as easy oxidation and dense interlayer stacking in a water/oxygen environment. At present, there is no comprehensive introduction to the research progress, advantages and disadvantages of MXenes in the field of flexible energy storage devices. Therefore, this review outlines the research progress of MXene-based nanocomposites (MXenes, MXenes/carbon, MXenes/metal oxide, MXenes/polymer) in the field of flexible energy storage electronic devices. This review summarizes the research progress of MXenes from three aspects: flexible capacitors, flexible batteries and flexible sensors. We compared MXenes with other two-dimensional materials in terms of synthesis methods and physical properties (such as conductivity and stress-strain properties), and discussed the problems and challenges faced by MXenes in the practical application of flexible devices. We also objectively discuss the development direction of MXenes in flexible energy storage devices.

Keywords: MXenes; Energy storage device; Two-dimensional material; Two-dimensional materials; Nanocomposite

\section{$\underline{\text { FULL TEXT }}$}

(C) 2021 The Authors. Published by ESG (www.electrochemsci.org). This article is an open access article distributed under the terms and conditions of the Creative Commons Attribution license (http://creativecommons.org/licenses/by/4.0/). 